\title{
Effects of feeding Saccharomyces cerevisiae fermentation products on the health of Holstein dairy calves following a lipopolysaccharide challenge
}

\author{
Rebecca N. Klopp, ${ }^{1} \odot$ Ilkyu Yoon, ${ }^{2} \odot$ Susan Eicher, ${ }^{3} \odot$ and Jacquelyn P. Boerman ${ }^{1 *} \odot$ \\ ${ }^{1}$ Department of Animal Sciences, Purdue University, West Lafayette, IN 47907 \\ 2Diamond V, Cedar Rapids, IA 52404 \\ ${ }^{3}$ USDA-ARS, Livestock Behavior Research Unit, West Lafayette, IN 47907
}

\section{ABSTRACT}

Before weaning, dairy calves are at high risk for illness, especially respiratory and digestive diseases, which reduces average daily gain, age at first calving, and first-lactation milk production. Although these illnesses are commonly treated with antibiotics, efforts are being made to reduce antibiotic use, due to concerns about antibiotic-resistant bacteria. The objective was to evaluate the effects of Saccharomyces cerevisiae fermentation products (SCFP) on the immune status of calves, following a lipopolysaccharide (LPS) challenge administered just before weaning. Thirty Holstein bull calves were blocked based on initial body weight and then assigned to 1 of 2 study treatments. The control group (CON) was fed a $24 \%$ crude protein: $17 \%$ fat milk replacer (MR) and calf starter with no SCFP added. The SCFP treatment was fed the same $24 \%$ crude protein:17\% fat MR with $1 \mathrm{~g} / \mathrm{d}$ of SmartCare (Diamond V) and calf starter with $0.8 \%$ NutriTek (Diamond V). SmartCare and NutriTek are both produced from anaerobic fermentation of $S$. cerevisiae. Calves were offered $2.84 \mathrm{~L}(12.5 \%$ solids) of MR twice daily at 0630 and $1630 \mathrm{~h}$ through d 51; from d 52 to 56 , calves were fed MR once daily at $0630 \mathrm{~h}$; and calves were weaned on $\mathrm{d}$ 57. Calves also received ad libitum access to a texturized calf starter and water. On d 50, a subset of calves ( $\mathrm{n}=20,10$ calves per treatment) were enrolled in an LPS challenge. At $-1.5,-0.5,0.5,1,1.5,2,2.5,3$, $3.5,4,5,6,7,8$, and $24 \mathrm{~h}$ relative to dosing with LPS, $20 \mathrm{~mL}$ of blood was collected, and rectal temperature and respiration rate were measured for each calf. Blood serum samples were analyzed for interleukin 6 , TNF- $\alpha$ (tumor necrosis factor- $\alpha$ ), interferon-gamma, haptoglobin, serum amyloid-A, fibrinogen, nonesterified

Received February 21, 2021.

Accepted October 13, 2021.

*Corresponding author: jboerma@purdue.edu fatty acid, cortisol, and glucose. This study observed increased concentrations of TNF- $\alpha$ at $1 \mathrm{~h}$ and $1.5 \mathrm{~h}$ and glucose at $0.5 \mathrm{~h}$ after dosing with LPS in SCFP calves compared with CON. Calves supplemented with SCFP also had an increase in respiration rate $0.5 \mathrm{~h}$ after dosing with LPS and reduced feed intake the day of the challenge compared with CON calves. These results suggest that dairy calves supplemented with SCFP exhibit an increased acute immune response, as observed by increased TNF- $\alpha$, glucose, and respiration rate immediately after dosing with LPS, compared with CON calves.

Key words: calf health, Saccharomyces cerevisiae fermentation products, lipopolysaccharide challenge

\section{INTRODUCTION}

Dairy calves in the United States have a high risk for morbidity before weaning, with 1 in 5 experiencing a respiratory disease and 1 in 4 a digestive illness, according to a nationwide survey of dairy farms (NAHMS, 2012). The most common way to treat these illnesses is with antibiotics. Of calves that are diagnosed with a respiratory disease, 9 in 10 are treated with antibiotics, and 7 in 10 calves diagnosed with digestive illnesses are treated with antibiotics (NAHMS, 2012). In animal agriculture, the use of antibiotics has come under scrutiny because of the increasing prevalence of antibiotic resistance of both human and animal pathogens (Langford et al., 2003; Loo et al., 2019), indicating a need for strategies to both improve calf health and reduce dependency on antibiotics.

Many different feed additives have been evaluated in dairy calves to reduce morbidity and improve health and growth, including Saccharomyces cerevisiae fermentation products (SCFP). When S. cerevisiae yeast is anaerobically fermented, amino acids, lipids, nucleotides, $\mathrm{B}$ vitamins, and organic acids are produced (Deters et al., 2018), and these are referred to as SCFP. Therefore, the effect of SCFP does not rely on the viability of live 
yeast. Studies suggest that SCFP may enhance health status through anti-inflammatory and antioxidant activities (Jensen et al., 2008b), via increased rumen development and reduced colonization of pathogenic bacteria (Brewer et al., 2014), priming the innate response before an immune challenge (Burdick Sanchez et al., 2020), and reducing the incidence of scouring (Alugongo et al., 2017; Harris et al., 2017), as well as increasing innate immune system cytokine production by peripheral blood mononuclear cells (Mahmoud et al., 2020).

The effects of SCFP on DMI and ADG have been reported in multiple studies. Research has reported positive effects on DMI (Lesmeister et al., 2004; Harris et al., 2017) as well as on ADG (Lesmeister et al., 2004; Harris et al., 2015) when calves are fed SCFP. In regard to the influence of SCFP on calf health, the most common health benefits are improved fecal score and reduced diarrhea (Magalhães et al., 2008; Alugongo et al., 2017). The rumen environment in calves can be a major indicator of health status. Reduction in ruminal microbial diversity within young calves has been linked to increased incidence of diarrhea (Xiao et al., 2016); SCFP may help improve microbial diversity and therefore reduce diarrhea. Additionally, in vitro, SCFP are capable of inhibiting pathogenic bacteria while promoting commensal bacteria (Jensen et al., 2008a), potentially indicating another mode of action for how SCFP reduces the incidence of diarrhea in calves.

Even though the effects of SCFP on calf health and performance have been widely researched, there are still more questions to explore regarding the specific mechanism behind the effects of SCFP on calf immunity. To evaluate this, calves' innate immune systems were tested using an LPS challenge model. The objective of this study was to evaluate the effects of SCFP on calves' immune status (temperature, respiration rate, metabolite, cytokine, and acute-phase protein analysis) following an LPS challenge that occurred before weaning. The hypothesis for this study was that all calves following the LPS dosing would experience an activated innate immune response, as evidenced by increased concentrations of cytokines and acute-phase proteins in circulation, as well as increases in temperature and respiration rate. However, the SCFP calves would have an elevated and rapid innate immune response and increased cytokine and heat production, compared with control calves. This acute immune response could neutralize foreign invaders more rapidly, leaving more energy available for growth and fewer negative effects from pathogens, which could explain the increased growth performance and health status observed in prior research when calves are fed SCFP.

\section{MATERIALS AND METHODS}

\section{Animals and Facility}

All animal procedures were reviewed and approved by the Institutional Animal Care and Use Committee at Purdue University (West Lafayette, IN; protocol no. 1808001783). Thirty Holstein bull calves, approximately $5 \pm 3$ (mean \pm SD) d of age, were received on September 13, 2019, from a dairy farm $55 \mathrm{~km}$ from the Purdue University Animal Sciences Research and Education Center Dairy Farm (West Lafayette, IN). Calves were transported to the Animal Sciences Research and Education Center by trailer $(2.3 \times 7.3$ $\mathrm{m}$; Wilson Trailer). Calves were housed in individual hutches (Calf-Tel) with a fenced-in outside area (3.5 $\times 1.2 \mathrm{~m}$ ). Individual hutches were bedded with wood shavings and re-bedded as needed.

\section{Study Treatments}

Upon arrival at the Purdue Animal Sciences Research and Education Center Dairy Farm, calves were assigned to 1 of 2 treatments $(\mathrm{n}=15$ each; Table 1$)$. The control (CON) treatment fed a $24 \%$ CP: $17 \%$ fat milk replacer (MR) and a texturized calf starter with no SCFP added. The SCFP treatment fed a $24 \%$ CP:17 $\%$ fat MR with $1 \mathrm{~g} / \mathrm{d}$ of SmartCare (Diamond V) and calf starter with $0.8 \%$ NutriTek (Diamond V). Calves were blocked into low BW (block $1, \mathrm{n}=10$ ), intermediate $\mathrm{BW}$ (block $2, \mathrm{n}=10$ ), or high $\mathrm{BW}$ (block $3, \mathrm{n}=$ $10)$ and then randomly assigned to treatment (CON or SCFP) within each block. Packed cell volume (PCV) was also measured upon arrival to determine the hydration status of each calf with all calves above $21 \%$ for packed cell volume.

Table 1. Chemical composition $( \pm \mathrm{SD})$ of milk replacer $(\mathrm{MR})$ and calf starter for control (CON; $\mathrm{n}=15)$ and Saccharomyces cerevisiae fermentation products (SCFP; $\mathrm{n}=15$ ) experimental treatment $\operatorname{diets}^{1}$

\begin{tabular}{lcrr}
\hline Item & MR & \multicolumn{1}{c}{$\begin{array}{c}\text { CON } \\
\text { starter }\end{array}$} & \multicolumn{1}{c}{$\begin{array}{c}\text { SCFP } \\
\text { starter }\end{array}$} \\
\hline Dry matter, \% & $96.6 \pm 0.1$ & $86.6 \pm 0.3$ & $86.6 \pm 0.5$ \\
CP, \% DM & $25.2 \pm 0.3$ & $23.7 \pm 2.2$ & $21.7 \pm 1.2$ \\
Fat, \% DM & $17.8 \pm 0.2$ & $3.6 \pm 0.2$ & $3.4 \pm 0.6$ \\
ADF, \% DM & - & $10.7 \pm 0.7$ & $10.3 \pm 0.5$ \\
aNDF, \% DM & - & $18.4 \pm 0.5$ & $19.0 \pm 1.1$ \\
Ash, \% DM & $8.7 \pm 0.1$ & $7.8 \pm 0.8$ & $7.3 \pm 0.5$ \\
\hline
\end{tabular}

${ }^{1} \mathrm{CON}$ (MR and calf starter with no SCFP added) or SCFP [MR with $1 \mathrm{~g} / \mathrm{d}$ of SmartCare (Diamond V) and calf starter with NutriTek (Diamond V)].

${ }^{2}$ Contained $0.8 \%$ NutriTek.

${ }^{3}$ Amylase-treated NDF. 


\section{Feeding Regimen}

Calves received $2.84 \mathrm{~L}$ of MR (12.5\% solids) twice daily at 0630 and $1630 \mathrm{~h}$ until d 51 of the study. From d 52 to 56 , calves were fed $2.84 \mathrm{~L}$ of MR once daily at $0630 \mathrm{~h}$ and were weaned on $\mathrm{d} 57$. If a calf refused more than $0.95 \mathrm{~L}$ of $\mathrm{MR}$, they were fed the remainder of the milk using an esophageal tube feeder (Nasco), to ensure sick calves that would not drink received sufficient nutrients and maintained hydration status. Refusals of MR less than $0.95 \mathrm{~L}$ were reported as a refusal. Calves also received ad libitum access to a texturized calf starter from d 1 to 56 .

\section{Lung Scanning}

On d 0, 28, and 49 (before LPS challenge), lung consolidation was evaluated for each calf using a portable ultrasonography machine (Ibex Pro, E.I. Medical Imagine) and a linear transducer (L6.2, E.I. Medical Imaging). Calves were restrained using a halter. The left and right lungs were scored separately on a scale of 1 to 4. A score of 1 indicates a normal lung with no lung consolidation (firming of the lungs) and no comet-tail artifacts (pleural roughening). A score of 2 indicates the presence of comet-tails but no lung consolidation. A score of 3 indicates lung consolidation that affects only 1 lobe. A score of 4 indicates lung consolidation that affects 2 or more lobes (modified from Ollivett and Buczinski, 2016).

\section{Lipopolysaccharide Challenge}

A subset of calves (20 total, 10 calves per treatment) were enrolled in an LPS challenge on d 50. Calves were enrolled in the challenge if they had not been previously treated for a respiratory illness, had no lung consolidation, and were consuming more than 900 $\mathrm{g}$ of starter daily. On d 49 (pre-challenge) and d 52 (post-challenge), $3 \mathrm{~mL}$ of blood was collected from the jugular vein of calves using an EDTA evacuated tube to quantify the cell types in blood, also known as complete blood counts, using a hematology analyzer (Genesis, Oxford Science). On d 49, calves were moved from their individual hutches to individual stalls in a tiestall barn at the Purdue Dairy Farm and restrained using halters. Calves were weighed (Tru-Test Inc.) to calculate LPS dose, scanned again for lung consolidation (Ibex Pro), and catheterized in the jugular vein using a 16-gauge $\times 7.5-\mathrm{cm}$ extended-use MilaCath (Mila International Inc.).

At $0830 \mathrm{~h}$ on d 50 of the study, 2 of the 20 calves received $0.125 \mu \mathrm{g} / \mathrm{kg}$ of BW of LPS from Escherichia coli
(O111:B4; Sigma L4391, Sigma Aldrich) intravenously. At $0930 \mathrm{~h}$, the remaining 18 calves received the same dose of LPS. Administration of LPS was staggered to ensure the dose was effective and to minimize any potential negative effects before dosing all 20 calves. Epinephrine, flunixin meglumine (Banamine, Merck Animal Health), and dexamethasone were available for medical intervention in case of an emergency. Blood was collected at $-1.5,-0.5,0.5,1,1.5,2,2.5,3,3.5,4$, $5,6,7,8$, and $24 \mathrm{~h}$ relative to dosing with LPS. At each blood collection, catheters were first flushed with $5 \mathrm{~mL}$ of saline $(0.9 \% \mathrm{NaCl})$, and then $20 \mathrm{~mL}$ of blood was collected, and catheters were flushed again with $5 \mathrm{~mL}$ of saline followed by $3 \mathrm{~mL}$ of heparinized saline $(2 \mathrm{~mL}$ of heparin $1,000 \mathrm{IU} / \mathrm{mL}$ in $1 \mathrm{~L}$ of saline). At the same time points that blood was collected, rectal temperature was measured using a digital thermometer (Vicks SpeedRead, Helen of Troy Ltd.), and respiration rate was determined by counting the number of breaths per minute. Blood was allowed to clot and then centrifuged at $3,100 \times g$ for $20 \mathrm{~min}$ at $4^{\circ} \mathrm{C}$. Serum was transferred to microcentrifuge tubes and frozen to $-20^{\circ} \mathrm{C}$ until further analysis.

Serum samples were sent to the University of Minnesota Cytokine Reference Laboratory (Minneapolis, MN) and were analyzed for IL-6, tumor necrosis factor- $\alpha(\mathrm{TNF}-\alpha)$, interferon-gamma (IFN- $\gamma$ ), haptoglobin (Hp), serum amyloid-A (SAA), and fibrinogen. Additional serum samples were analyzed using commercial kits for nonesterified fatty acid [NEFA; Wako HR series NEFA-HR(2); FujiFilm Wako Diagnostics U.S.A. Corp.], cortisol (EKC31009; Biomatik Corp.), and glucose (P7119; Sigma Aldrich). Interassay coefficients of variation were $5.45 \%, 4.65 \%, 13.11 \%$ for NEFA, glucose, and cortisol, respectively. Intra-assay coefficients of variation were $3.65 \%, 2.67 \%, 4.24 \%$ for NEFA, glucose, and cortisol, respectively.

\section{Statistical Analyses}

A power analysis was performed to calculate the sample size for a primary outcome variable, TNF- $\alpha$ concentrations, following LPS challenge in calves. Based on data from Benjamin et al. (2016), with 95\% confidence and $80 \%$ power, 9 animals per treatment group were needed to detect differences. To account for potential calf mortality during the LPS challenge, 10 animals per treatment group were enrolled in the challenge. Data were analyzed as a completely randomized design using the Mixed Procedure of SAS version 9.4 (SAS Institute Inc.) with repeated measures, using a first-order autoregressive structure $[\mathrm{AR}(1)]$ based on minimizing Akaike information criterion and Bayes- 
ian information criterion. To identify serum analysis outliers, a Cook's distance cutoff of 0.2 was set. One data point was removed from the NEFA analysis, and 3 data points were removed from the cortisol analysis. Measurements for serum analytes, rectal temperature, and respiration rate at all time points were analyzed for normality using a Shapiro-Wilk test, and data were normally distributed. One calf on the SCFP treatment died during the LPS challenge, and that calf's data were removed from analysis. The experimental unit was individual calf $(\mathrm{n}=19)$. The fixed effects included treatment $\left[\mathrm{T}_{\mathrm{i}} ; \operatorname{CON}(\mathrm{n}=10)\right.$, and $\left.\operatorname{SCFP}(\mathrm{n}=9)\right]$, time point $\left(\mathrm{P}_{\mathrm{j}} ;-1.5,-0.5,0.5,1,1.5,2,2.5,3,3.5\right.$, $4,5,6,7,8$, and $24 \mathrm{~h}$ relative to LPS dosing for rectal temperature, respiratory rate, metabolites, cytokines, and acute-phase proteins; 1 and 2 for complete blood count analysis before and after LPS challenge; $-1,0,1$, $2,3,4$ for intake in relation to LPS challenge), and the interactions between treatment and time point $\left[\mathrm{TP}_{(\mathrm{ij})}\right]$. The random effect was calf nested within treatment $\left[\mathrm{C}_{\mathrm{k}(\mathrm{i})}\right]$. The model was represented as follows:

$$
\mathrm{Y}_{\mathrm{ijk}}=\mu+\mathrm{T}_{\mathrm{i}}+\mathrm{P}_{\mathrm{j}}+\mathrm{TP}_{(\mathrm{ij})}+\mathrm{C}_{\mathrm{k}(\mathrm{i})}+\mathrm{e}_{\mathrm{ijk}},
$$

where $Y_{\mathrm{ijk}}$ is the response variable, $\mu$ is the overall mean, and $e_{i j k}$ is the error. Continuous response variables included rectal temperature, respiratory rate, intake in relation to LPS challenge, complete blood count analysis, NEFA, TNF- $\alpha$, IL- 6 , IFN- $\gamma$, Hp, SAA, fibrinogen, cortisol, and glucose. A $P$-value $\leq 0.05$ was determined to be statistically significant, and a $P$-value $>0.05$ and $\leq 0.10$ was determined to be a statistical tendency. When $\mathrm{TP}_{(\mathrm{ij})}$ was statistically significant or a tendency $(P \leq 0.10)$, the slice option was used to evaluate the acute (0.5 to $4 \mathrm{~h}$ post-LPS dosing) treatment effects within time point. A Bonferroni adjustment was applied to the slice option to decrease the probability of type 1 error.

\section{RESULTS}

One calf died during the LPS challenge, even after emergency medical intervention. That calf was a part of the SCFP treatment and died $2 \mathrm{~h}$ after dosing.

\section{Rectal Temperature, Respiratory Rate, and Intake}

A time point effect was observed for rectal temperature $(P<0.0001)$, which increased after dosing with LPS, peaked around $3.5 \mathrm{~h}$ after dosing, and then continued to decrease until $24 \mathrm{~h}$ after dosing (Figure 1). A tendency for a treatment by time point interaction was observed for respiration rate $(P=0.07$; Figure 2 ). At $0.5 \mathrm{~h}$, SCFP calves had an increased respiration rate $[103$ vs. 80 breaths $/ \mathrm{min} \pm 5.4(\mathrm{SEM}) ; P=0.02]$ compared with CON calves.

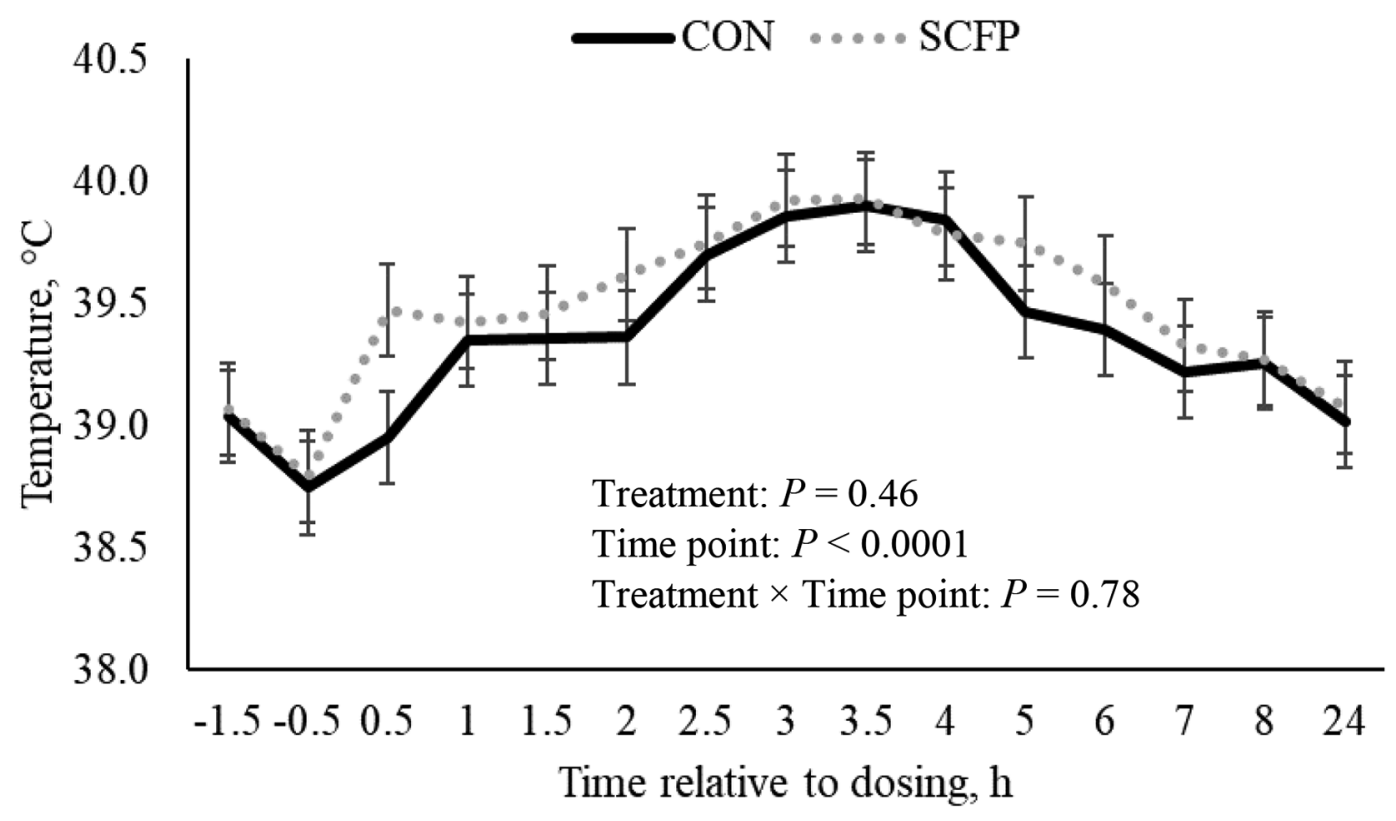

Figure 1. Rectal temperature by treatment in relation to LPS challenge dosing. Calves were assigned to 1 of 2 treatments as follows: CON [24\% CP:17\% fat milk replacer (MR) and calf starter with no SCFP added; $\mathrm{n}=10]$ or SCFP $[24 \% \mathrm{CP}: 17 \%$ fat MR with $1 \mathrm{~g} / \mathrm{d}$ of SmartCare (Diamond V) and calf starter with $0.8 \%$ NutriTek (Diamond V); $\mathrm{n}=9]$. CON = control diet; SCFP = Saccharomyces cerevisiae fermentation product diet. Error bars represent SEM. 
We found no starter intake differences between treatments the day before the LPS challenge, with SCFP calves consuming 1,093 g and CON calves consuming $1,267 \mathrm{~g}(P=0.54)$. On the day of the LPS challenge, SCFP calves consumed $761 \mathrm{~g} /$ calf less starter compared with the CON calves $(P=0.002$; Table 2$)$. No intake differences based on treatment after the day of the LPS challenge were observed $(P>0.29)$.

\section{Complete Blood Count Pre-Challenge and Post-Challenge}

When comparing blood counts pre-challenge (d 49) and post-challenge (d 52), a time point effect occurred for white blood cells (WBC; $10^{3} / \mu \mathrm{L}$ ), lymphocytes $\left(10^{3} / \mu \mathrm{L}\right)$, eosinophils $\left(10^{3} / \mu \mathrm{L}\right)$, neutrophil/lymphocyte ratio, basophils $\left(10^{3} / \mu \mathrm{L}\right)$, and red blood cells (RBC; $10^{6} / \mu \mathrm{L}$; all $P<0.01$; Table 3$)$. Compared with before the LPS challenge, WBC, lymphocytes, and RBC increased after the LPS challenge (all $P<0.001$ ). Eosinophils, basophils, and the neutrophil/lymphocyte ratio decreased after the LPS challenge (all $P<0.05$ ). No treatment or treatment by time point interactions occurred for complete blood counts $(P \geq 0.20)$.

\section{Blood Parameters During LPS Challenge}

A treatment by time point interaction was observed for TNF- $\alpha$ (Figure 3; $P=0.005$ ) and glucose (Figure
Table 2. Starter intake, in grams, of control diet $(\mathrm{CON} ; \mathrm{n}=10)$ and diet with Saccharomyces cerevisiae fermentation products (SCFP; $\mathrm{n}=$ 9) in relation to LPS challenge (d 50)

\begin{tabular}{lrrrc}
\hline & \multicolumn{2}{c}{ Treatment $^{2}$} & & \\
\cline { 2 - 3 } $\begin{array}{l}\text { Day relative to } \\
\text { LPS challenge }\end{array}$ & CON & SCFP & SEM & $P$-value \\
\hline-1 & 1,267 & 1,093 & 198 & 0.54 \\
0 & 1,328 & 567 & 148 & 0.002 \\
1 & 1,469 & 1,346 & 109 & 0.42 \\
2 & 1,884 & 1,891 & 89 & 0.95 \\
3 & 1,866 & 1,819 & 90 & 0.71 \\
4 & 1,890 & 1,871 & 94 & 0.89 \\
\hline
\end{tabular}

${ }^{1}$ Calves received $0.125 \mu \mathrm{g} / \mathrm{kg}$ of $\mathrm{BW}$ of LPS from Escherichia coli (O111:B4; Sigma L4391, Sigma Aldrich) intravenously.

${ }^{2} \mathrm{CON}[24 \% \mathrm{CP}: 17 \%$ fat milk replacer (MR) and calf starter with no SCFP added] or SCFP [24\% CP:17\% fat MR with $1 \mathrm{~g} / \mathrm{d}$ of SmartCare (Diamond V) and calf starter with $0.8 \%$ NutriTek (Diamond V)].

4; $P=0.02$ ). At 1 and $1.5 \mathrm{~h}$ after dosing with LPS, SCFP calves had increased concentrations of TNF- $\alpha$ in circulation compared with $\mathrm{CON}$ calves $(P \leq 0.02)$. At $0.5 \mathrm{~h}$ after dosing with LPS, SCFP calves had increased concentrations of serum glucose compared with $\mathrm{CON}$ calves $(P=0.02)$. A treatment by time point tendency was observed for SAA, where SCFP calves had greater SAA concentrations at various time points after dosing with LPS $(P=0.10$; Table 4$)$. No treatment by time point interactions were observed for NEFA, IL6 , IFN- $\gamma$, Hp, fibrinogen, or cortisol $(P>0.10)$. No treatment differences were observed for NEFA, TNF- $\alpha$,

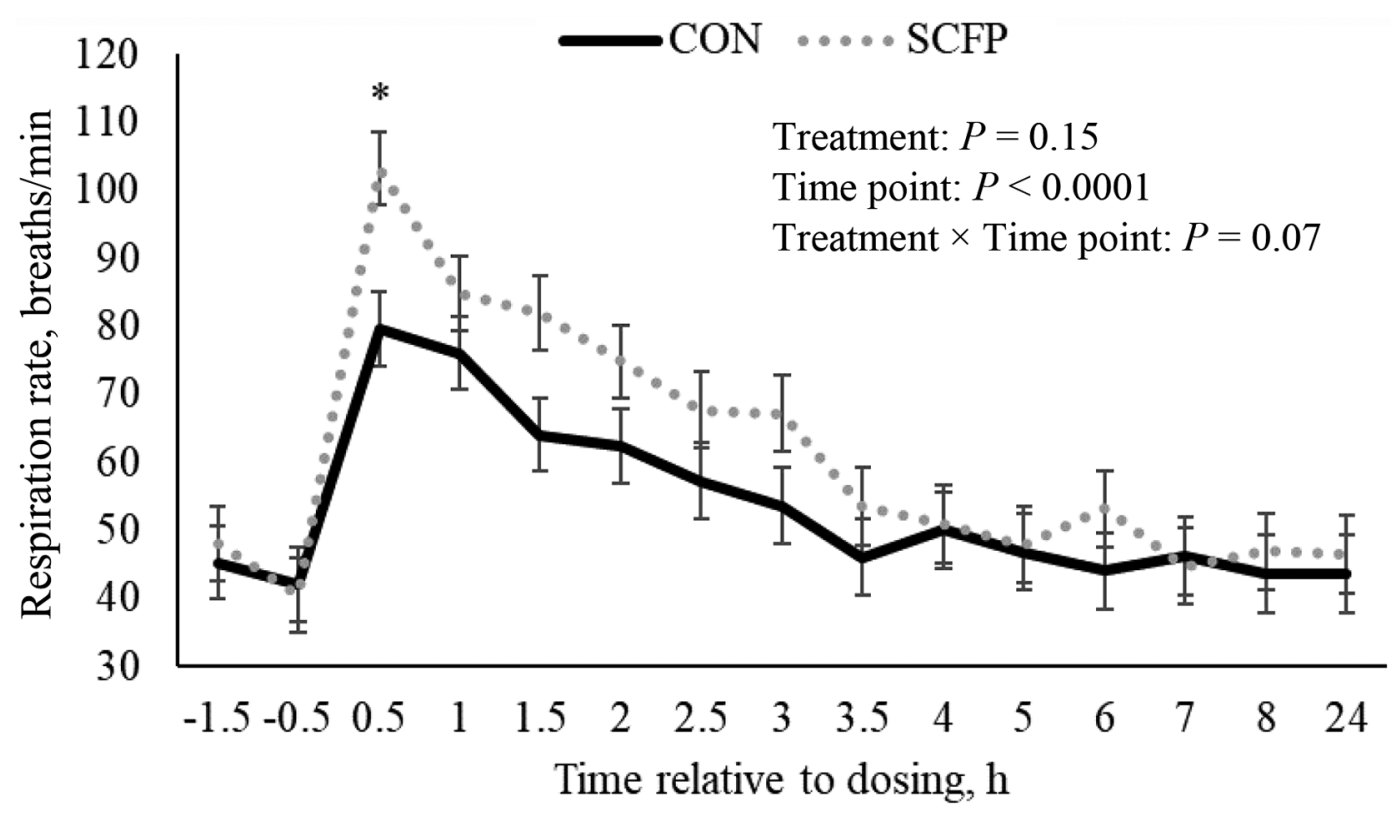

Figure 2. Respiration rate by treatment in relation to LPS challenge dosing. Calves were assigned to 1 of 2 treatments as follows: CON [24\% CP:17\% fat milk replacer (MR) and calf starter with no SCFP added; $\mathrm{n}=10]$ or SCFP $[24 \%$ CP:17\% fat MR with $1 \mathrm{~g} / \mathrm{d}$ of SmartCare (Diamond V) and calf starter with $0.8 \%$ NutriTek (Diamond V); $\mathrm{n}=9]$. CON $=$ control diet; SCFP $=$ Saccharomyces cerevisiae fermentation product diet. ${ }^{*}$ Indicates a significant difference $(P \leq 0.05)$ between treatments. Error bars represent SEM. 
IL-6, IFN- $\gamma$, Hp, fibrinogen, cortisol, or glucose based on overall treatment averages $(P>0.10)$. However, a treatment tendency was observed for SAA $(P=0.10)$. A time point effect was detected for NEFA, TNF- $\alpha$, IL-6, IFN- $\gamma$, SAA, fibrinogen, cortisol, and glucose $(P$ $<0.05)$.

\section{DISCUSSION}

The overall findings from this study were that SCFP calves had a greater acute immune response after dosing with LPS; however, this increased response only lasted approximately $2 \mathrm{~h}$. This was evident by increased respiration rate, $\mathrm{TNF}-\alpha$, and glucose within the first 2 $\mathrm{h}$ after dosing with LPS and reduced intake the day of the challenge.

An LPS challenge is designed to mimic a bacterial infection, causing the innate immune system to be activated. However, during an LPS challenge the animal is not actually exposed to bacteria, just to LPS, a component in the cell wall of gram-negative bacteria; therefore the immune response is acute and short-lived. When the immune system is activated, a variety of inflammatory mediators (cytokines and acute-phase proteins) are stimulated, which can result in an increase in body temperature (Dinarello, 1996); this explains why an increase in rectal temperature shortly after dosing with LPS was observed in all calves in this study. Temperature increased quickly, peaked $3.5 \mathrm{~h}$ after dosing with LPS, and then returned to values similar to pre-challenge $7 \mathrm{~h}$ after dosing. A study looking at the acute immune response of purebred Angus steers after an LPS challenge observed a similar rectal temperature response, where values increased shortly after dosing with LPS, peaked at $4.5 \mathrm{~h}$, and were still elevated at $8 \mathrm{~h}$ after dosing compared with pre-challenge values (Carroll et al., 2009). This same increased temperature response shortly after dosing with LPS has been observed in other studies as well (Steiger et al., 1999; Waldron et al., 2003). The current study, however, did not observe a treatment by time point interaction for temperature, dissimilar to a previous studies evaluating SCFP. Burdick Sanchez et al. (2020) fed SCFP to weaned beef steers $(274 \pm 1.9 \mathrm{~kg} \mathrm{BW})$ and observed a treatment by time interaction for rectal temperature. They observed increased rectal temperature with SCFP-fed calves at $1 \mathrm{~h}$ after dosing with LPS $(0.25 \mu \mathrm{g} / \mathrm{kg}$ of BW $)$. They observed a treatment difference in rectal temperature again at 6 to $11 \mathrm{~h}, 13 \mathrm{~h}, 15$ to $20 \mathrm{~h}$, and 22 to $24 \mathrm{~h}$ after the challenge, although the temperatures during

Table 3. Complete blood count of control $(\mathrm{CON} ; \mathrm{n}=10)$ and Saccharomyces cerevisiae fermentation product $(\mathrm{SCFP} ; \mathrm{n}=9)$ diets fed to calves pre- (d 49) and post- (d 52) LPS challenge (d 50) ${ }^{1}$

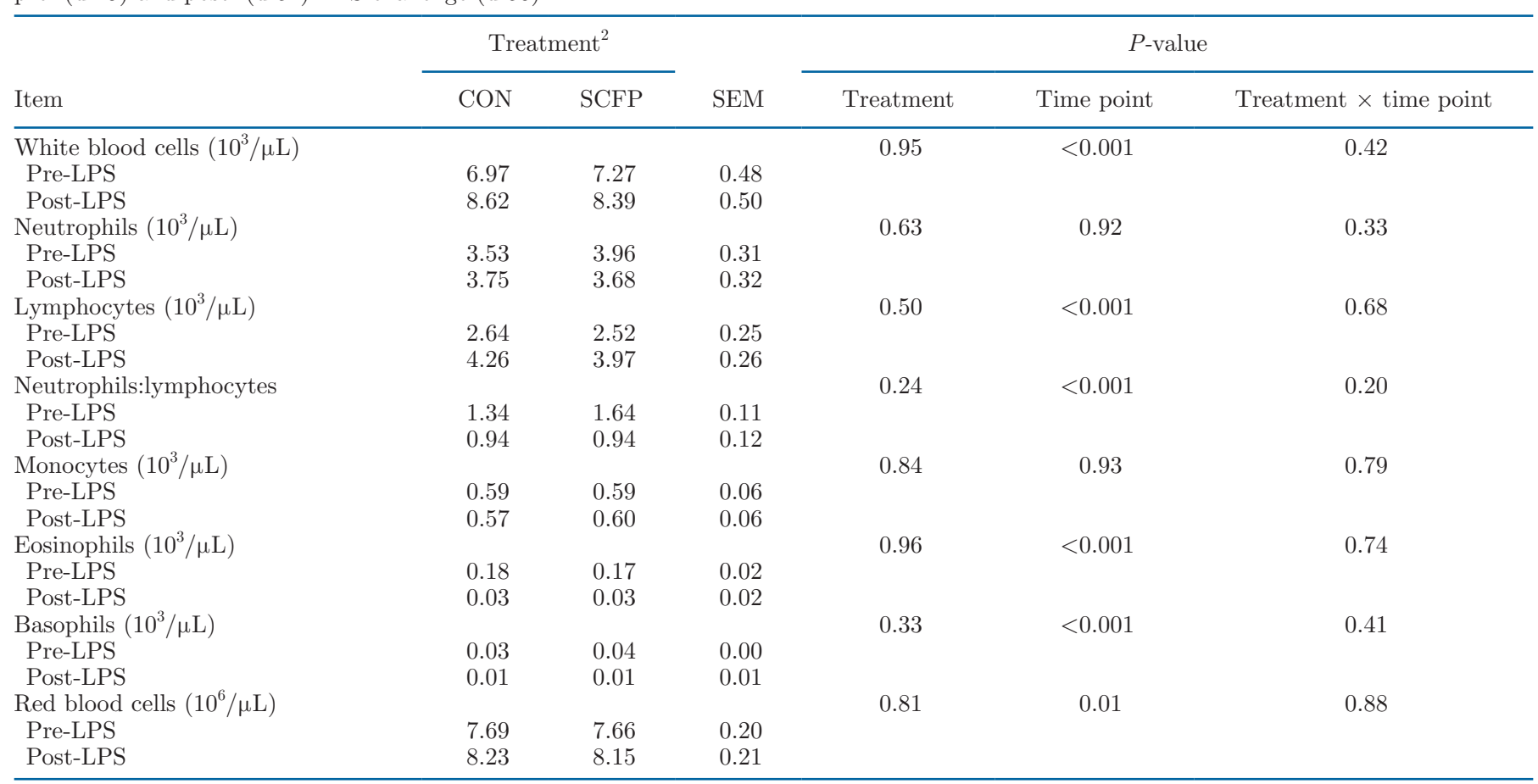

${ }^{1}$ Calves received $0.125 \mu \mathrm{g} / \mathrm{kg}$ of BW of LPS from Escherichia coli (O111:B4; Sigma L4391, Sigma Aldrich) intravenously.

${ }^{2} \mathrm{CON}[24 \% \mathrm{CP}: 17 \%$ fat milk replacer (MR) and calf starter with no SCFP added] or SCFP [24\% CP:17\% fat MR with $1 \mathrm{~g} / \mathrm{d}$ of SmartCare (Diamond V) and calf starter with $0.8 \%$ NutriTek (Diamond V)]. 


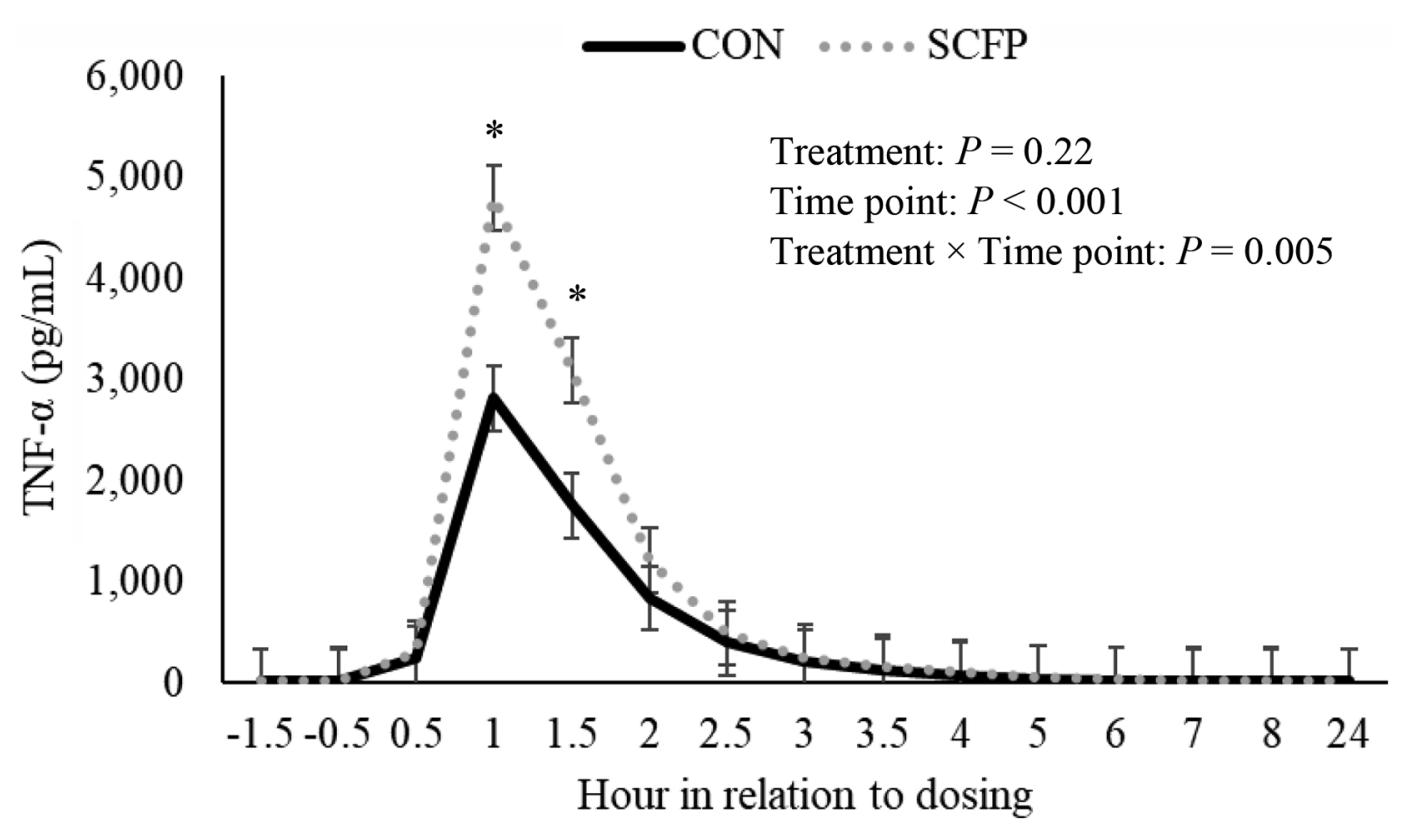

Figure 3. TNF- $\alpha$ concentrations by treatment in relation to LPS dosing. Calves were assigned to 1 of 2 treatments as follows: CON [24\% CP: $17 \%$ fat milk replacer (MR) and calf starter with no SCFP added; $\mathrm{n}=10]$ or SCFP $[24 \%$ CP:17\% fat MR with $1 \mathrm{~g} / \mathrm{d}$ of SmartCare (Diamond $\mathrm{V}$ ) and calf starter with $0.8 \%$ NutriTek (Diamond V); $\mathrm{n}=9$ ]. CON $=$ control diet; SCFP $=$ Saccharomyces cerevisiae fermentation product diet. *Indicates a significant difference $(P \leq 0.05)$ between treatments. Error bars represent SEM.

these time points were equal to or lower than basal temperature before the challenge.

During periods of stress, such as an immune challenge, the adrenal gland will release adrenaline and increase respiration rates. In cattle, the major anaphylactic shock organ is the lung (Eyre et al., 1973). The current study observed an increase in respiratory rate for all calves, which peaked $0.5 \mathrm{~h}$ after dosing with LPS and returned to pre-challenge values $3.5 \mathrm{~h}$ after. Carroll et al. (2009) also observed similar respiration rate responses in Angus steers, which had a sharp increase $0.5 \mathrm{~h}$ after dosing with LPS and then returned to pre-challenge rates at $2.5 \mathrm{~h}$. The current study also observed that SCFP calves had an increase in respiration rate compared with $\mathrm{CON}$ calves, $0.5 \mathrm{~h}$ after dosing with LPS. This suggests that SCFP-fed calves responded to the LPS challenge more quickly and at a greater rate compared with $\mathrm{CON}$ calves. However, the increased response was not prolonged, as no differences in respiration rate were observed for any time points after $3.5 \mathrm{~h}$ following LPS dosing. Burdick Sanchez et al. (2020) did not report respiration rates; however, they did report sickness behavior scores, which are based on both behavior and respiration rate, with 1 being normal behavior and 4 being laying on side with labored breathing and frothing at the mouth. They reported that SCFP calves tended to have lower sickness behavior scores compared with $\mathrm{CON}$ calves at 1 to 2 $\mathrm{h}$ and $3.5 \mathrm{~h}$ after dosing with LPS; they did mention that overall sickness behavior scores were relatively low in both treatments. The discrepancy about how SCFP affects respiration rates between Burdick Sanchez et al. (2020) and the current study could be due to the age, breed, diet, LPS dose, and length of treatment of the calves. In the current study, calves were younger and smaller when they experienced the LPS challenge compared with the calves in Burdick Sanchez et al. (2020).

Even though the current study did not observe increased rectal temperature in SCFP calves following LPS challenge, this response has been observed previously (Burdick Sanchez et al., 2020). However, the current study did observe an increased respiration rate in SCFP calves compared with CON. An increase in respiration rate at the same time as increased temperature is reasonable, as it has been reported that the 2 are correlated (Scharf et al., 2010). These responses following an LPS challenge would suggest an increased acute immune response. Increase in body temperature has been shown to reduce the growth of bacteria and increase animal survivability, as increased body temperature reduces the ability of pathogenic bacteria to grow (Kluger and Rothenburg, 1979; Kluger et al., 1998). The lack of a treatment effect or a treatment by time point interaction for rectal temperature in the current study may be due to the timing and quantity of time points analyzed and the briefness of the acute immune 


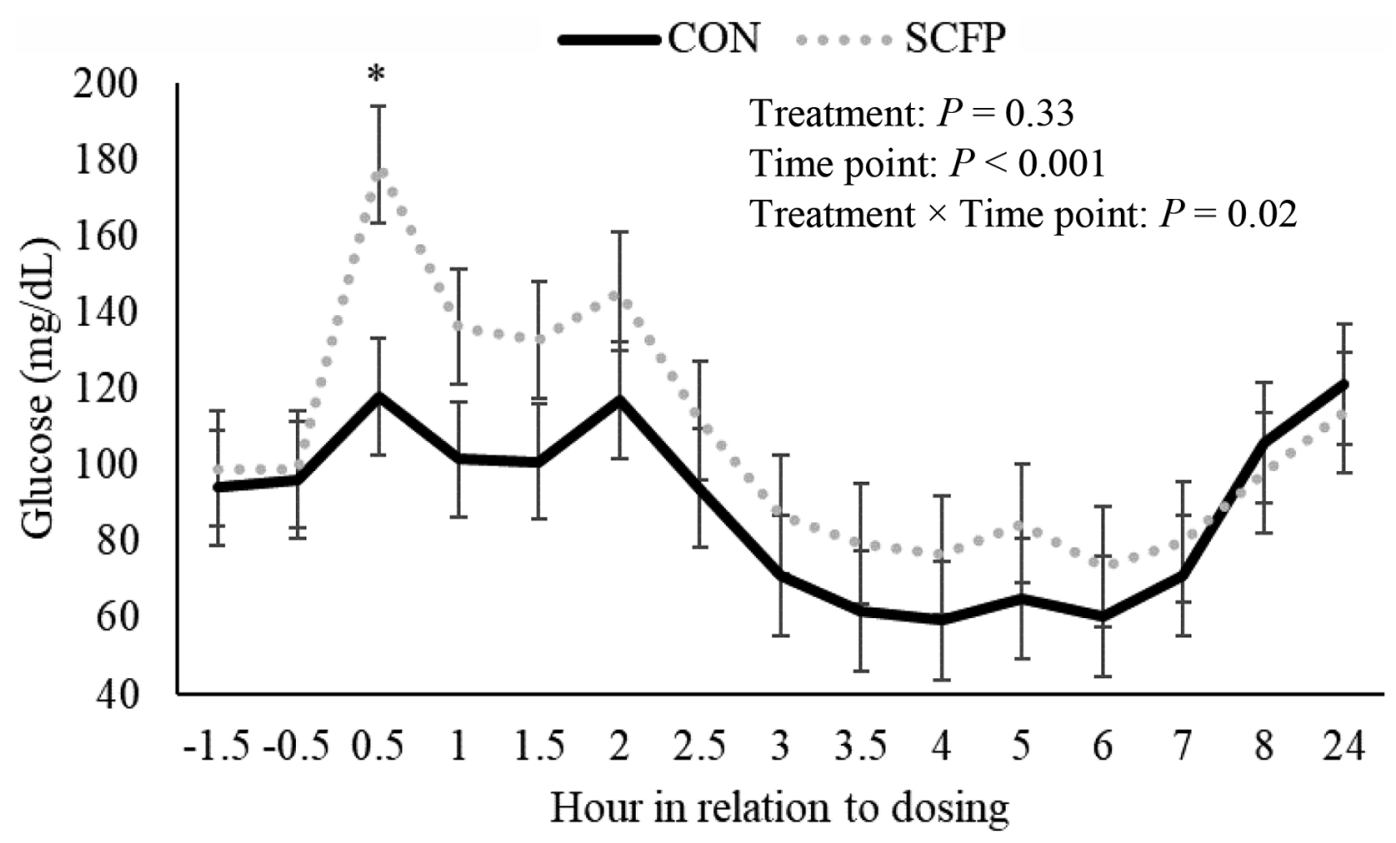

Figure 4. Glucose concentrations by treatment in relation to LPS dosing. Calves were assigned to 1 of 2 treatments as follows: CON [24\% CP: $17 \%$ fat milk replacer (MR) and calf starter with no SCFP added; $n=10]$ or SCFP $[24 \%$ CP:17\% fat MR with $1 \mathrm{~g} / \mathrm{d}$ of SmartCare (Diamond $\mathrm{V}$ ) and calf starter with $0.8 \%$ NutriTek (Diamond V); $\mathrm{n}=9]$. CON $=$ control diet; SCFP $=$ Saccharomyces cerevisiae fermentation product diet. *Indicates a significant difference $(P \leq 0.05)$ between treatments. Error bars represent SEM.

response. However, it is worth noting that a prolonged increase in body temperature can have a negative effect on energy stores in the body and could be detrimental to survivability (Carroll and Forsberg, 2007).

On the day of the LPS challenge, SCFP calves consumed less starter compared with the CON calves. This difference in intake, based on treatment, was not observed before the challenge (d 49) or in the days after the challenge (d 51-54). Anorexia (decreased appetite) is a defense mechanism used by hosts to reduce feed intake and thus reduce the entry of the pathogen across the lumen into circulation (Exton, 1997). To the best of the authors' knowledge, no other study has reported reduced intake in SCFP calves compared with CON during an LPS challenge. However, other studies have reported reduced intakes in general in cattle during an LPS challenge (Werling et al., 1996; Steiger et al., 1999; Waldron et al., 2003). Also, acute immune responseinduced stress can often lead to a reduction in feed intake (Carroll and Forsberg, 2007). In this study, the

Table 4. Serum analysis of metabolites, cytokines, and acute-phase proteins of control (CON; n = 10) and Saccharomyces cerevisiae fermentation product $(\mathrm{SCFP} ; \mathrm{n}=9)$ diets fed to calves during LPS challenge $(\mathrm{d} 50)^{1}$

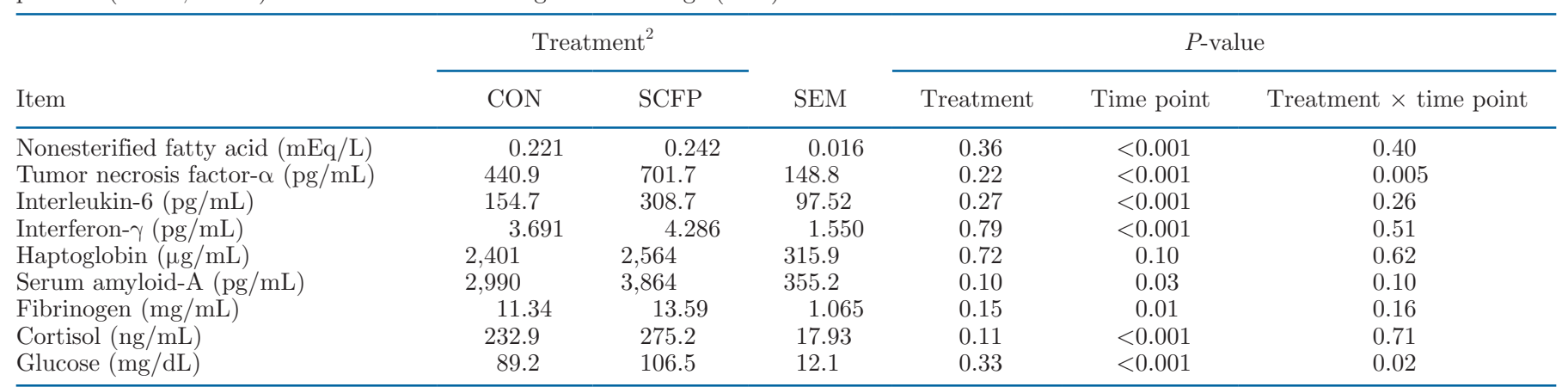

${ }^{1}$ Calves received $0.125 \mu \mathrm{g} / \mathrm{kg}$ of BW of LPS from Escherichia coli (O111:B4; Sigma L4391, Sigma Aldrich) intravenously.

${ }^{2} \mathrm{CON}$ [24\% CP:17\% fat milk replacer (MR) and calf starter with no SCFP added] or SCFP [24\% CP:17\% fat MR with $1 \mathrm{~g} / \mathrm{d}$ of SmartCare (Diamond V) and calf starter with $0.8 \%$ NutriTek (Diamond V)]. 
observed response was a sharp increase in the stress hormone cortisol in circulation $0.5 \mathrm{~h}$ after dosing with LPS, which progressively decreased over the next $24 \mathrm{~h}$.

White blood cells are the immune cells of the body and include eosinophils, basophils, monocytes, lymphocytes, and neutrophils. The $\mathrm{RBC}$ help to regulate the homeostasis of chemokines (Anderson et al., 2018), which are chemoattractant cytokines that attract immune cells to the site of infection (Delves and Roitt, 2000). Therefore, observing an increase in immune cells and RBC in circulation after an immune challenge is typical, as the body calls upon them to prompt an effective immune response. Research in weaned pigs using the LPS challenge model observed a steady increase in WBC from 2 to $24 \mathrm{~h}$ after dosing with LPS (Burdick Sanchez et al., 2018). Another study reported WBC counts in nonlactating dairy cows exposed to different amounts of LPS from 0 to $144 \mathrm{~h}$ after dosing (Jacobsen et al., 2005). They observed elevated WBC from 24 to $96 \mathrm{~h}$ after dosing when cows received 100 or 1,000 ng of LPS $/ \mathrm{kg}$ of BW, which aligns with the results of increased WBC at $48 \mathrm{~h}$ after LPS dosing in this study.

When the immune system is activated, TNF- $\alpha$, a proinflammatory cytokine, is secreted. Therefore, it is expected to see an increase in circulating TNF- $\alpha$ during an LPS challenge (Carroll et al., 2009; Benjamin et al., 2016; Burdick Sanchez et al., 2020). The cytokine TNF- $\alpha$ signals inflammation, which leads to heat production by affecting the thermoregulatory centers in the brain (Dinarello, 1996). The current study observed a treatment by time point interaction for TNF- $\alpha$, where SCFP calves had increased TNF- $\alpha$ concentrations soon after dosing with LPS. Although we did not see a treatment or treatment by time point effect for rectal temperature, a time point effect was observed for rectal temperature, which increased in calves soon after dosing with LPS. Burdick Sanchez and colleagues (2020) reported increased body temperature in SCFP calves but decreased $\mathrm{TNF}-\alpha$, and were therefore unclear what was causing the increased body temperature. However, their reported TNF- $\alpha$ concentrations were relatively low compared with this study and what others have reported (Waldron et al., 2003; Carroll et al., 2009; Benjamin et al., 2016). This variability in TNF- $\alpha$ concentrations is likely due to differences in the dose of LPS used across studies, as well as the health status and age of animals. Even though IL-6 concentrations were not statistically different between treatments in our study, likely due to the large variation observed, SCFP calves did have numerically increased IL-6 compared with CON. Increases in IL-6 combined with elevated TNF- $\alpha$, SAA, and respiration rate in SCFP calves supports that SCFP calves had a different innate immune response compared with CON calves following the LPS challenge.

Shortly after dosing, an increase in circulating glucose concentrations in SCFP calves was observed. Glucose is the main energy source for the immune system (Wolowczuk et al., 2008), so this increase in glucose could be the body's response to an activated immune system and ensuring enough energy to elicit an immune response to the foreign invader (Lang et al., 1985). This study did observe a treatment by time point interaction for glucose: SCFP calves had numerically increased glucose concentrations for most of the post-challenge period. This numerical increase in glucose observed in the SCFP calves compared with the CON, coupled with an increase in respiration rate and $\mathrm{TNF}-\alpha$, as well as reduced intake, all suggest an increased acute immune response by the SCFP calves in response to the LPS challenge. Burdick Sanchez et al. (2020) also reported greater glucose in SCFP claves at various time points during the post-challenge period, which was believed to be a glucose-sparing effect by the SCFP calves, but this is an area that requires further research.

Further research is needed to understand the mechanism behind how SCFP influences the innate immune system. Although not specifically addressed here, another area where knowledge is limited is the question of at what point an increase in the responsiveness of the immune system becomes detrimental to the growth and survivability of the animal, by diverting excessive amounts of energy to the immune system.

\section{CONCLUSIONS}

In summary, during an LPS challenge, calves supplemented with SCFP experienced a greater innate immune response. This was evident by an increase in circulating TNF- $\alpha$, which promotes inflammation and protects the body from foreign invaders. The stress of an activated immune system led to increased respiration rates and a reduction in feed intakes. Calves on the SCFP treatment also had increased circulating glucose directly following the LPS challenge. These results suggest that supplementing calves with SCFP increases the acute immune response of calves. More research is needed to determine whether SCFP improves health outcomes in animals challenged with specific pathogens that elicit an immune response.

\section{ACKNOWLEDGMENTS}

Conflict of interest: IY is employed by Diamond V (Cedar Rapids, IA). This research was funded by Diamond V. The authors have not stated any other con- 
flicts of interest. The authors acknowledge the Purdue Animal Sciences graduate students N. Briggs, T. Hurst, and C. McCabe (Purdue University, West Lafayette, IN) for their help and dedication throughout the study. The authors also thank Purdue Animal Sciences undergraduates K. Dager, K. Harmes, M. Keirn, E. Ludwick, K. Teeple, and E. Thrasher, for their contributions to the project, and M. Grott and the remainder of the staff at the Animal Sciences Research and Education Center (West Lafayette, IN) for making this work possible.

\section{REFERENCES}

Alugongo, G. M., J. X. Xiao, Y. H. Chung, S. Z. Dong, S. L. Li, I. Yoon, Z. H. Wu, and Z. J. Cao. 2017. Effects of Saccharomyces cerevisiae fermentation products on dairy calves: Performance and health. J. Dairy Sci. 100:1189-1199. https://doi.org/10.3168/jds .2016-11399.

Anderson, H. L., I. E. Brodsky, and N. S. Mangalmurti. 2018. The evolving erythrocyte: Red blood cells as modulators of innate immunity. J. Immunol. 201:1343-1351. https://doi.org/10.4049/ jimmunol.1800565.

Benjamin, A. L., F. T. Korkmaz, T. H. Elsasser, and D. E. Kerr. 2016. Neonatal lipopolysaccharide exposure does not diminish the innate immune response to a subsequent lipopolysaccharide challenge in Holstein bull calves. J. Dairy Sci. 99:5750-5763. https://doi.org/ 10.3168/jds.2015-10804.

Brewer, M. T., K. L. Anderson, I. Yoon, M. F. Scott, and S. A. Carlson. 2014. Amelioration of salmonellosis in pre-weaned dairy calves fed Saccharomyces cerevisiae fermentation products in feed and milk replacer. Vet. Microbiol. 172:248-255. https://doi.org/10 .1016 /j.vetmic.2014.05.026.

Burdick Sanchez, N. C., J. A. Carroll, P. R. Broadway, B. E. Bass, and J. W. Frank. 2018. Modulation of the acute phase response following a lipopolysaccharide challenge in pigs supplemented with an all-natural Saccharomyces cerevisiae fermentation product. Livest. Sci. 208:1-4. https://doi.org/10.1016/j.livsci.2017.11.022.

Burdick Sanchez, N. C., J. A. Carroll, P. R. Broadway, T. S. Edrington, I. Yoon, and C. R. Belknap. 2020. Some aspects of the acute phase immune response to a lipopolysaccharide (LPS) challenge are mitigated by supplementation with a Saccharomyces cerevisiae fermentation product in weaned beef calves. Transl. Anim. Sci. 4:txaa156. https://doi.org/10.1093/tas/txaa156.

Carroll, J. A., and N. E. Forsberg. 2007. Influence of stress and nutrition on cattle immunity. Vet. Clin. North Am. Food Anim. Pract. 23:105-149.

Carroll, J. A., R. R. Reuter, C. C. Chase Jr., S. W. Coleman, D. G. Riley, D. E. Spiers, J. D. Arthington, and M. L. Galyean. 2009. Profile of the bovine acute-phase response following an intravenous bolus-dose lipopolysaccharide challenge. Innate Immun. 15:81-89. https://doi.org/10.1177/1753425908099170.

Delves, P. J., and I. M. Roitt. 2000. The immune system. N. Engl. J. Med. 343:37-49. https://doi.org/10.1056/NEJM200007063430107.

Deters, E. L., R. S. Stokes, O. Genther-Schroeder, and S. L. Hansen. 2018. Effects of a Saccharomyces cerevisiae fermentation product in receiving diets of newly weaned beef steers. II. Digestibility and response to a vaccination challenge. J. Anim. Sci. 96:3906-3915.

Dinarello, C. A. 1996. Thermoregulation and the pathogenesis of fever. Infect. Dis. Clin. North Am. 10:433-449. https://doi.org/10.1016/ S0891-5520(05)70306-8.

Exton, M. S. 1997. Infection-induced anorexia: Active host defence strategy. Appetite 29:369-383. https://doi.org/10.1006/appe.1997 .0116 .
Eyre, P., A. J. Lewis, and P. W. Wells. 1973. Acute systemic anaphylaxis in the calf. Br. J. Pharmacol. 47:504-516. https://doi.org/10 $.1111 /$ j.1476-5381.1973.tb08182.x.

Harris, T. L., Y. Liang, M. D. Sellers, C. R. Nightingale, K. P. Sharon, J. A. Carroll, I. Yoon, M. F. Scott, and M. A. Ballou. 2015. Influences of SmartCare in milk replacer and XPC in calf starter on the performance and health of pre-weaning Holstein calves challenged orally with an opportunistic infection with Citrobacter freundii. J. Dairy Sci. 98(Suppl. 2):763. (Abstr.)

Harris, T. L., Y. Liang, K. P. Sharon, M. D. Sellers, I. Yoon, M. F. Scott, J. A. Carroll, and M. A. Ballou. 2017. Influence of Saccharomyces cerevisiae fermentation products, SmartCare in milk replacer and original XPC in calf starter, on the performance and health of preweaned Holstein calves challenged with Salmonella enterica serotype Typhimurium. J. Dairy Sci. 100:7154-7164. https://doi .org/10.3168/jds.2016-12509.

Jacobsen, S., T. Toelboell, and P. H. Andersen. 2005. Dose dependency and individual variability in selected clinical, haematological and blood biochemical responses after systemic lipopolysaccharide challenge in cattle. Vet. Res. 36:167-178. https://doi.org/10.1051/ vetres:2004062.

Jensen, G. S., K. M. Patterson, and I. Yoon. 2008a. Nutritional yeast culture has specific anti-microbial properties without affecting healthy flora. Preliminary results. J. Anim. Feed Sci. 17:247-252. https://doi.org/10.22358/jafs/66604/2008.

Jensen, G. S., K. M. Patterson, and I. Yoon. 2008b. Yeast culture has anti-inflammatory effects and specifically activates NK cells. Comp. Immunol. Microbiol. Infect. Dis. 31:487-500. https://doi .org/10.1016/j.cimid.2007.08.005.

Kluger, M. J., W. Kozak, C. A. Conn, L. R. Leon, and D. Z. Soszynski. 1998. Role of fever in disease. Ann. N. Y. Acad. Sci. 856:224-233. https://doi.org/10.1111/j.1749-6632.1998.tb08329.x.

Kluger, M. J., and B. A. Rothenburg. 1979. Fever and reduced iron: Their interaction as a host defense response to bacterial infection. Science 203:374-376. https://doi.org/10.1126/science.760197.

Lang, C. H., G. J. Bagby, and J. J. Spitzer. 1985. Glucose kinetics and body temperature after lethal and nonlethal doses of endotoxin. Am. J. Physiol. 248:R471-R478. https://doi.org/10.1152/ajpregu .1985.248.4.R471.

Langford, F. M., D. M. Weary, and L. Fisher. 2003. Antibiotic resistance in gut bacteria from dairy calves: A dose response to the level of antibiotics fed in milk. J. Dairy Sci. 86:3963-3966. https:/ /doi.org/10.3168/jds.S0022-0302(03)74006-5.

Lesmeister, K. E., A. J. Heinrichs, and M. T. Gabler. 2004. Effects of supplemental yeast (Saccharomyces cerevisiae) culture on rumen development, growth characteristics, and blood parameters in neonatal dairy calves. J. Dairy Sci. 87:1832-1839. https://doi.org/10 .3168/jds.S0022-0302(04)73340-8.

Loo, E., K. S. Lai, and R. Mansor. 2019. Antimicrobial usage and resistance in dairy cattle production. Pages $7-21$ in Veterinary Medicine and Pharmaceuticals. S. O. Bekoe, M. Saravanan, R. K. Adosraku, and P. L. Ramkumar, ed. Intech Open.

Magalhães, V. J. A., F. Susca, F. S. Lima, A. F. Branco, I. Yoon, and J. E. P. Santos. 2008. Effect of feeding yeast culture on performance, health, and immunocompetence of dairy calves. J. Dairy Sci. 91:1497-1509. https://doi.org/10.3168/jds.2007-0582.

Mahmoud, A. H. A., J. R. Slate, S. Hong, I. Yoon, and J. L. McGill. 2020. Supplementing a Saccharomyces cerevisiae fermentation product modulates innate immune function and ameliorates bovine respiratory syncytial virus infection in neonatal calves. J. Anim. Sci. 98:skaa252. https://doi.org/10.1093/jas/skaa252.

NAHMS. 2012. Dairy Heifer Raiser, 2011. USDA-APHIS-VS, Washington, DC.

Ollivett, T. L., and S. Buczinski. 2016. On-farm use of ultrasonography for bovine respiratory disease. Vet. Clin. North Am. Food Anim. Pract. 32:19-35.

Scharf, B., J. A. Carroll, D. G. Riley, C. C. Chase Jr., S. W. Coleman, D. H. Keisler, R. L. Weaber, and D. E. Spiers. 2010. Evaluation of physiological and blood serum differences in heat-tolerant (Ro- 
mosinuano) and heat-susceptible (Angus) Bos taurus cattle during controlled heat challenge. J. Anim. Sci. 88:2321-2336. https://doi .org/10.2527/jas.2009-2551.

Steiger, M., M. Senn, G. Altreuther, D. Werling, F. Sutter, M. Kreuzer, and W. Langhans. 1999. Effect of a prolonged low-dose lipopolysaccharide infusion on feed intake and metabolism in heifers. J. Anim. Sci. 77:2523-2532. https://doi.org/10.2527/1999.7792523x.

Waldron, M. R., T. Nishida, B. J. Nonnecke, and T. R. Overton. 2003 Effect of lipopolysaccharide on indices of peripheral and hepatic metabolism in lactating cows. J. Dairy Sci. 86:3447-3459. https:// doi.org/10.3168/jds.S0022-0302(03)73949-6.

Werling, D., F. Sutter, M. Arnold, G. Kun, P. C. J. Tooten, E. Gruys, M. Kreuzer, and W. Langhans. 1996. Characterisation of the acute phase response of heifers to a prolonged low dose infusion of lipopolysaccharide. Res. Vet. Sci. 61:252-257. https://doi.org/10 1016/S0034-5288(96)90073-9.

Wolowczuk, I., C. Verwaerde, O. Viltart, A. Delanoye, M. Delacre, B. Pot, and C. Grangette. 2008. Feeding our immune system: Impact on metabolism. Clin. Dev. Immunol. 2008:639803. https://doi.org/ $10.1155 / 2008 / 639803$.

Xiao, J. X., G. M. Alugongo, R. Chung, S. Z. Dong, S. L. Li, I. Yoon, Z. H. Wu, and Z. J. Cao. 2016. Effects of Saccharomyces cerevisiae fermentation products on dairy calves: Ruminal fermentation, gastrointestinal morphology, and microbial community. J. Dairy Sci. 99:5401-5412. https://doi.org/10.3168/jds.2015-10563.

\section{ORCIDS}

Rebecca N. Klopp ํ https://orcid.org/0000-0001-8485-6831

Ilkyu Yoon ( https://orcid.org/0000-0003-1891-1585

Susan Eicher $\odot$ https://orcid.org/0000-0001-5832-4049

Jacquelyn P. Boerman ( https://orcid.org/0000-0002-0336-8295 